\title{
No socioeconomic inequalities in ovarian cancer survival within two randomised clinical trials
}

M E Abdel-Rahman ${ }^{1}$, J Butler ${ }^{2}$, M R Sydes ${ }^{3}$, M K B Parmar ${ }^{3}$, E Gordon ${ }^{4}$, P Harper ${ }^{5,9}$, C Williams ${ }^{6,9}$, A Crook ${ }^{3,9}$, J Sandercock ${ }^{7,9}$, A M Swart ${ }^{8,9}$, B Rachet ${ }^{1}$ and M P Coleman ${ }^{*, 1}$ on behalf of the ICON2 and ICON3 investigators ${ }^{9}$

${ }^{1}$ Cancer Research UK Cancer Survival Group, Department of Non-Communicable Disease Epidemiology, London School of Hygiene and Tropical Medicine, Keppel Street, London WC1E 7HT, UK; ${ }^{2}$ Royal Marsden Hospital, Fulham Road, London SW3 6JJ, UK; ${ }^{3}$ MRC Clinical Trials Unit at UCL, Aviation House, 125 Kingsway, London WC2B 6NH, UK; ${ }^{4}$ National Cancer Intelligence Centre, Office for National Statistics, Cardiff Road, Newport NP10 8XG, UK; ${ }^{5}$ London Oncology Clinic, 95 Harley Street, London W1G 6AF, UK; ${ }^{6}$ Bristol Haematology and Oncology Centre, University Hospitals Bristol, Horfield Road, Bristol BS2 8ED, UK; ${ }^{7}$ Metasurv wordpress and ${ }^{8}$ Norwich Medical School, University of East Anglia, Norwich Research Park NR4 7TJ, UK

Background: Ovarian cancer is the leading cause of death among cancers of the female genital tract, with poor outcomes despite chemotherapy. There was a persistent socioeconomic gradient in 1-year survival in England and Wales for more than 3 decades (1971-2001). Inequalities in 5-year survival persisted for more than 20 years but have been smaller for women diagnosed around 2000. We explored one possible explanation.

Methods: We analysed data on 1406 women diagnosed with ovarian cancer during 1991-1998 and recruited to one of two randomised clinical trials. In the second International Collaborative Ovarian Neoplasm (ICON2) trial, women diagnosed between 1991 and 1996 were randomised to receive either the three-drug combination cyclophosphamide, doxorubicin and cisplatin (CAP) or single-agent carboplatin given at optimal dose. In the ICON3 trial, women diagnosed during 1995-1998 were randomised to receive either the same treatments as ICON2, or paclitaxel plus carboplatin. Relative survival at 1, 5 and 10 years was estimated for women in five categories of socioeconomic deprivation. The excess hazard of death over and above background mortality was estimated by fitting multivariable regression models with Poisson error structure and a dedicated link function in a generalised linear model framework, adjusting for the duration of follow-up and the confounding effects of age, Federation of Gynecology and Obstetrics (FIGO) stage and calendar period.

Results: Unlike women with ovarian cancer in the general population, no statistically significant socioeconomic gradient was seen for women with ovarian cancer treated in the two randomised controlled trials. The deprivation gap in 1-year relative survival in the general population was statistically significant at $-6.7 \%(95 \% \mathrm{Cl}(-8.1,-5.3))$, compared with $-3.6 \%(95 \% \mathrm{Cl}(-10.4,+3.2))$ in the trial population.

Conclusions: Although ovarian cancer survival is significantly lower among poor women than rich women in England and Wales, there was no evidence of an association between socioeconomic deprivation and survival among women with ovarian cancer who were treated and followed up consistently in two well-conducted randomised controlled trials. We conclude that the persistent socioeconomic gradient in survival among women with ovarian cancer, at least for 1-year survival, may be due to differences in access to treatment and standards of care.

*Correspondence: Professor MP Coleman; E-mail: michel.coleman@|shtm.ac.uk

${ }^{9} \mathrm{~A}$ list of centres and clinicians participating in ICON2 and ICON3 in England and Wales is provided before References.

Received 23 January 2014; revised 9 May 2014; accepted 11 May 2014; published online 10 June 2014

(c) 2014 Cancer Research UK. All rights reserved 0007-0920/14 
Socioeconomic disparities in survival are observed for many adult cancers in England and Wales (Coleman et al, 2004; Rachet et al, 2008) and in other parts of the world (Woods et al, 2006; Jeffreys et al, 2009). For ovarian cancer in England and Wales, throughout the 31-year period 1971-2001, 1-year survival in the most deprived group of women was 7-9\% lower than in the most affluent group (Coleman et al, 1999; Cooper et al, 2008). For 5-year survival, this 'deprivation gap' was 2-3\% for women diagnosed up to 1990; however, it then declined, and no deprivation gap in 5-year survival was observed for women diagnosed around 2000.

Socioeconomic disparities in ovarian cancer survival are only partially explained by differences in the extent of disease at diagnosis and differences in treatment (Woods et al, 2006). In the United States of America, ovarian cancer survival is substantially lower in Blacks than Whites (Barnholtz-Sloan et al, 2002). Differences in ovarian cancer survival may be explained by differences in tumour biology and morphology, delay in diagnosis, the quality of the staging procedures or differences in treatment (Maringe et al, 2012).

One way to assess whether socioeconomic differences in survival are related to treatment is to measure the deprivation gap in a setting that is not directly affected by other aspects of the healthcare system (Nur et al, 2008, 2012). Patients recruited to clinical trials are randomly assigned to specific treatment regimes regardless of their socioeconomic status, or indeed of any other potentially confounding variable. Close adherence to trial protocol ensures equality of access to and compliance with treatment among all socioeconomic groups. The impact of age and stage at diagnosis on socioeconomic differentials can be quantified in the analysis; however, only a small proportion of women with ovarian cancer are recruited to clinical trials. By contrast, population-based studies are very large because they include all patients, thus providing a public health perspective on socioeconomic inequalities in outcome; however, data on stage and treatment are rarely available for all patients (Walters et al, 2013). This makes it more difficult to control for early diagnosis, stage and treatment when quantifying socioeconomic differences in survival in the general population.

The objective of this study was to investigate socioeconomic differences in ovarian cancer survival among women recruited to randomised trials and to compare these differences with those observed among women with ovarian cancer in the general population. If the 'deprivation gap' in survival in the general population also arises in a trial, then it is more likely to be because of biological or environmental factors than to differences in treatment. If, on the other hand, a socioeconomic survival gradient is not observed in a clinical trial, after adjustment for known prognostic factors, then the deprivation gap in survival in the general population is more likely to be because of differences in treatment, since that is the one factor that is reliably and equally accessible to all socioeconomic groups within a randomised trial.

\section{PATIENTS AND METHODS}

Study population. This study used data on women from England and Wales recruited to two international ovarian cancer trials, linking data from the MRC Trials Unit, the National Cancer Registry and other sources.

As part of the International Collaborative Ovarian Neoplasm (ICON) studies, the Medical Research Council (MRC) Cancer Trial Office in Cambridge, UK (now the MRC Clinical Trials Unit, London) participated in two trials, ICON2 and ICON3, which compared the outcome from various chemotherapy treatments after definitive local surgery. The main eligibility criterion was that the woman was assessed by the clinician as requiring chemotherapy and being fit to receive it. The trials included women in both early and advanced stages of disease. The women's socioeconomic status was not recorded at recruitment.

ICON2 recruited women between January 1991 and July 1996 and compared two internationally standard regimens in a $1: 1$ ratio: the three-drug combination of cyclophosphamide, doxorubicin and cisplatin (CAP) against single-agent carboplatin given at an optimal dose. For ICON3, women were recruited between February 1995 and October 1998 and randomised 2:1 to a choice of the ICON2 treatments, or randomisation within the ICON2 protocol, or to paclitaxel plus carboplatin.

Randomisation was performed by straight minimisation, stratifying by (i) centre, (ii) disease stage (using the International Federation of Gynecology and Obstetrics (FIGO) stage) and (iii) residual bulk of disease, plus for ICON3 only (iv) time since surgery and (v) choice of control regimen. This method ensures balance between treatment groups for the specified factors (Pocock and Simon, 1975). Both trials aimed to compare progression-free and overall survival between the treatment regimens. Detailed descriptions of ICON2 (Parmar et al, 2002) and ICON3 (Parmar et al, 1998) have been published. The results showed no evidence of differences in survival between the various treatment regimens (ICON2: hazard ratio $1.00,95 \%$ CI $0.86-1.16, P$-value 0.98 ; ICON3: hazard ratio $0.98,95 \%$ CI $0.87-1.10, P$-value 0.74$)$. For the analyses reported here, socioeconomic differences in survival among the women recruited to each trial were therefore analysed without reference to the various treatment protocols. The key is that all the women in the trials received treatments which, we now know, were either identical, if they were in the same arm of the trial, or equivalent with respect to outcome, if in different arms.

The MRC Clinical Trials Unit provided information on the date of birth, the tumour, FIGO stage, date of randomisation, type of treatment and the outcome for all women recruited to ICON2 and ICON3. For women resident in England and Wales at diagnosis, the Office for National Statistics (ONS) provided information on vital status at 31 December 2007 - the last day of follow-up for this study - and the date of death for women who had died, by linkage between the National Cancer Registry and the National Health Service Central Register (NHSCR).

An ecological deprivation score was assigned to each woman, based on the characteristics of the Lower Super Output Area (LSOA) in which she was resident at diagnosis, and the year of diagnosis. The 34378 LSOAs in England and Wales are small areas (mean population 1500) covering the entire country and for which detailed data on a wide range of routinely collected variables, such as income, employment and housing, are available to characterise the level of deprivation of the residents. The Carstairs score (Carstairs, 1995), derived from the 1991 Census, was used for women diagnosed up to 1995 . For those diagnosed in 1996 or later, the income domain score of the Indices of Multiple Deprivation (IMD2004; Neighbourhood Renewal Unit, 2004) was used. LSOA deprivation scores were grouped into five categories, from the most affluent (1) to the most deprived (5), based on quintiles of the national distribution. Women with ovarian cancer were assigned to the deprivation category of their LSOA of residence, using their postcode and a combined historic file of over 2 million unique postcodes, linked to geographic area codes for each calendar year. For a given geographic unit in England and Wales in a given calendar period, the deprivation gap in survival is similar for all measures of deprivation (Woods et al, 2005).

A total of 1406 women recorded in the trial databases as resident in England or Wales were considered for analysis: 272 in ICON2 and 1134 in ICON3. Of these, 1290 (92\%; 242 in ICON2, 1048 in ICON3) were analysed after the following exclusions (Figure 1): (i) 73 women who could not be linked to a cancer registration record and thus to information on their vital status; (ii) 2 women with incomplete information on deprivation; (iii) 41 women with either no date of diagnosis (18), unknown vital status 

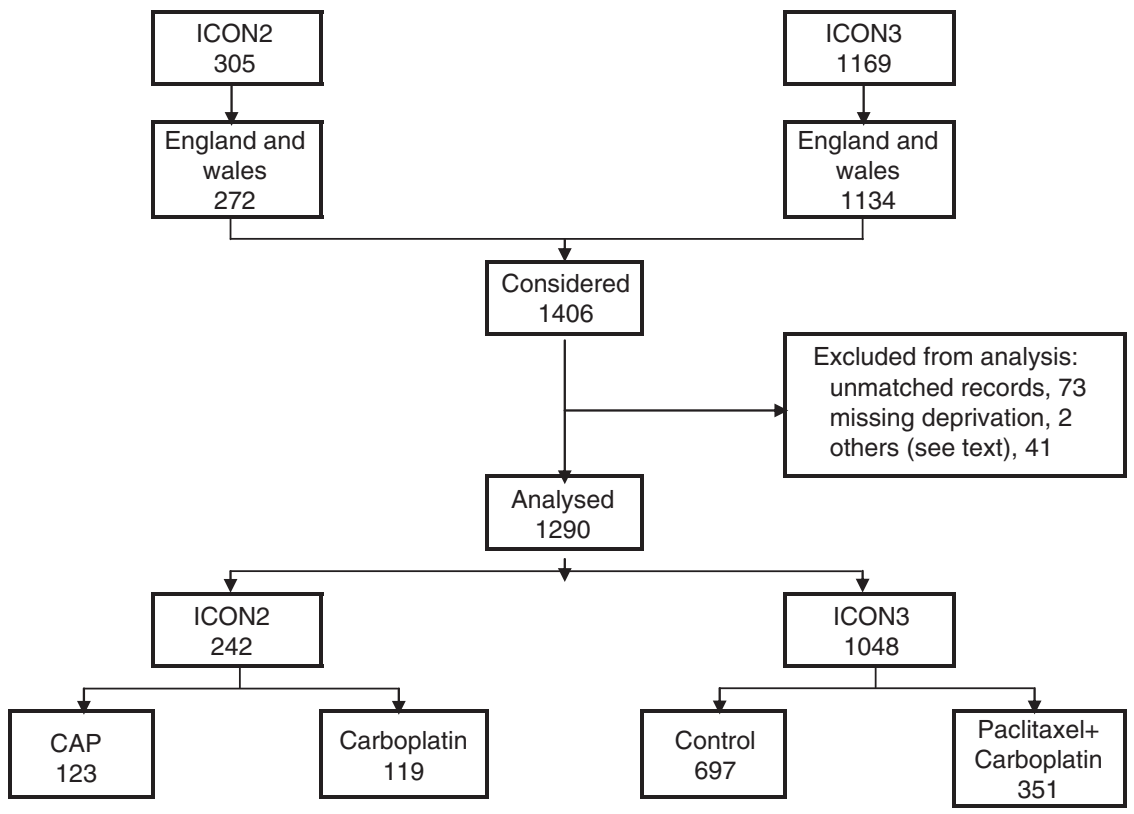

Figure 1 . The study population.

(2), synchronous tumours (3), metastasis to the ovary from another primary site (12) or not recorded as resident in England or Wales in the National Cancer Registry (6).

This research was approved by the Ethics Committee of the London School of Hygiene and Tropical Medicine. Appropriate ethical approvals were originally given to both ICON2 and ICON3 protocols, and approval to flag the patients for their vital status in the NHSCR was obtained from ONS.

Statistical analyses. Survival time was measured in days between the exact date of randomisation during the period 1991-1998 and the date of last vital status (the earlier of the date of death and 31 December 2007), and recorded in years (days/365.25).

Cumulative all-cause and relative survival up to 5 years after randomisation was estimated for each deprivation category. Survival probabilities were estimated at 3-month intervals for the first 6 months, then 6-month intervals up to 2 years and at annual intervals thereafter. Relative survival was estimated with the maximum likelihood approach for individual records (Estève et al, 1990), using the strel program (Cancer Research UK Cancer Survival Group, 2010) in Stata version 10 (StataCorp, 2006; College Station, TX, USA).

Relative survival (Ederer et al, 1961) may be interpreted as the survival from cancer after correction for other causes of death. It is defined as the ratio of the survival observed in the cancer patients and the survival that would have been expected had they been subjected only to the mortality rates observed in the general population (background mortality; Coleman et al, 1999).

Background mortality was taken from national life tables (Cancer Research UK Cancer Survival Group, 2004) incorporating all-cause death rates for women in England and Wales by single year of age (up to 99 years), single calendar year and deprivation category. Life tables were constructed from the numbers of deaths and the census populations by age and LSOA for 1991 and 2001, and smoothed using a Poisson regression model with spline functions. Intercensal life tables were interpolated linearly. Life tables for 2006 and 2007 could not be constructed because the relevant data (deaths during 2007-2008) were unavailable, so life tables for 2005 were used for these years without extrapolation. Survival gradients were estimated with variance-weighted linear regression (Grizzle et al, 1969) across all five categories of deprivation. The deprivation gap was taken as the absolute difference between the fitted relative survival estimates for the most affluent and the most deprived groups of women. A negative value is used to indicate that survival was lower for the most deprived group than the most affluent group.

We compared survival in the trial population with survival for all women diagnosed with an invasive primary ovarian malignancy in England and Wales during the same calendar period as the trial recruitment, also followed up to 31 December 2007. The deprivation gap was estimated in an identical manner for women in the trials and, separately, for all women registered with ovarian cancer in England and Wales during 1991-1998.

To estimate variations in survival due to deprivation, multivariable regression models were fitted in a Generalised Linear Model framework, with Poisson error structure and a dedicated link function (Dickman et al, 2004), adjusting for duration of follow-up, age, FIGO stage and period of diagnosis. The excess hazard of death over and above the background mortality in each deprivation category was modelled up to 5 years after randomisation. The excess hazard was assumed to be constant within each of a series of pre-specified time intervals since randomisation (Estève et al, 1990): 3-month intervals for the first 6 months, 6-month intervals up to 2 years and annual intervals thereafter. Estimation was based on exact survival times using grouped data (Dickman et al, 2004).

The initial model included the main effects of follow-up time and deprivation. Age at randomisation (under 55, 55-65, over 65 years), calendar period at randomisation (1991-1995, 1996-1999) and FIGO stage (I-IV) were successively included in the model. For each variable, time-dependent effects were tested separately by the introduction of interactions with time. Interactions between other factors were similarly tested. The likelihood ratio test $(P$ value $<0.05$ ) was used to assess improvement in model fit by the addition of other variables and interaction terms. Deviance statistics (McCullagh and Nelder, 1999) and residual plots were used as measures of goodness of fit.

Excess hazard ratios (EHRs), with their 95\% confidence intervals, are presented as the ratio of the excess hazard of death within 5 years of diagnosis in a given deprivation category relative to the most affluent category (reference group), after controlling for other factors included in the model. 


\section{RESULTS}

Women recruited to the ICON trials were evenly distributed across the five deprivation categories (18-21\%; Table 1), very similar to the pattern for all women diagnosed with ovarian cancer in England and Wales during 1991-1998 (18-21\%). Most women in the trials were under the age of 65 years, with a median age of 58 years, compared with a median of 65 years for all women with ovarian cancer in England and Wales. Almost two-thirds of women $(63 \%)$ were recruited with stage III disease, with little variation between socioeconomic groups (Table 1 ).

By the end of the follow-up, some 10-16 years after recruitment, 1086 (84\%) of the women had died; the remaining 204 women were censored from the analysis at study closure on 31 December 2007.

For women in the trials, 1-year survival was $77-78 \%$ in the three more affluent categories and $74-75 \%$ for the two most deprived groups (Table 2, Figure 2). One-year survival was $12-17 \%$ (mean $14.3 \%$ ) higher than for women in the same deprivation category in the general population during the same period, regardless of disease stage or treatment plan. For women in the trial, the fitted deprivation gap in 1-year survival was negative at $-4 \%$ (95\% CI $(-10,+3))$, whereas the deprivation gap in the general population was wider, and statistically significant: $-7 \%$ (95\% CI $(-8,-5))$.

Overall 5-year survival for women in the trials was $29.1 \%(95 \%$ CI $26.5-31.6 \%$ ) some $4 \%$ lower than for unselected women with ovarian cancer in the general population during the same period 33.5\% (33.0-34.0\%; Table 2, Figure 2). There was no consistent pattern by deprivation category, and the fitted deprivation gap was $-4 \%$ ( -12 to $+3 \%)$. The deprivation gap in survival in the general population was smaller: $-1 \%(-3$ to $0 \%)$.

Ten-year survival was only $19.5 \%(17.2-21.9 \%)$ for women in the trials, some $9 \%$ lower than for all women with ovarian cancer in the general population $(28.5 \%, 28.0-29.0 \%)$. There was no strong evidence of a deprivation gradient in 10-year survival, either among women in the trials $(1.2 \%,-5.5$ to $+7.9 \%)$, or among women with ovarian cancer in the general population $(0 \%,-1.1$ to $+1.9 \%)$.

The socioeconomic gradient in the excess hazard of death within 5 years of diagnosis was small $(1.17$; 95\% CI $0.95-1.44$; $P$-value 0.391; Table 3).

The excess hazard was higher for women aged 55-65 and those over 65 years at diagnosis than for women aged less than 55 (reference category; EHR 1.25, 95\% CI 1.1-1.5 for both groups; Table 3). The excess hazard was lower for women randomised during 1996-1999 than for those who were randomised on average about 5 years earlier, during 1991-1995 (EHR 0.85, 95\% CI 0.740.98 ): this represents a $15 \%$ reduction among patients diagnosed over that 5 -year period in the excess hazard of death within 5 years of diagnosis. The excess hazard also shows a marked and progressive increase across the four categories of FIGO stage at diagnosis (up to 4.3-fold for FIGO stage IV). All three effects are to be expected, and they largely explain even the modest deprivation gradient in the overall excess hazard (Table 3). Interaction of

Table 1. Distribution (no. and \%) of women recruited to ICON2 and ICON3 trials of ovarian cancer during 1991-1998 by deprivation category

Deprivation category

\begin{tabular}{|c|c|c|c|c|c|c|c|c|c|c|c|c|}
\hline & \multicolumn{10}{|c|}{ Deprivation category } & & \\
\hline & \multicolumn{2}{|c|}{ Affluent } & \multicolumn{2}{|c|}{2} & \multicolumn{2}{|c|}{3} & \multicolumn{2}{|c|}{4} & \multicolumn{2}{|c|}{ Deprived } & \multicolumn{2}{|c|}{ All women } \\
\hline & No. & $\%$ & No. & $\%$ & No. & $\%$ & No. & $\%$ & No. & $\%$ & No. & $\%$ \\
\hline \multicolumn{13}{|l|}{ Trial } \\
\hline ICON2 & 43 & 17.8 & 56 & 23.1 & 49 & 20.2 & 53 & 21.9 & 41 & 16.9 & 242 & 100.0 \\
\hline ICON3 & 230 & 21.9 & 188 & 17.9 & 223 & 21.3 & 212 & 20.2 & 195 & 18.6 & 1048 & 100.0 \\
\hline \multicolumn{13}{|l|}{ Treatment } \\
\hline ICON2: CAP & 18 & 14.6 & 38 & 30.9 & 24 & 19.5 & 26 & 21.1 & 17 & 13.8 & 123 & 100.0 \\
\hline Carboplatin & 25 & 21.0 & 18 & 15.1 & 25 & 21.0 & 27 & 22.7 & 24 & 20.2 & 119 & 100.0 \\
\hline ICON3: Control & 149 & 21.4 & 130 & 18.7 & 146 & 20.9 & 146 & 20.9 & 126 & 18.1 & 697 & 100.0 \\
\hline Paclitaxel & 81 & 23.1 & 58 & 16.5 & 77 & 21.9 & 66 & 18.8 & 69 & 19.7 & 351 & 100.0 \\
\hline \multicolumn{13}{|l|}{ Age (years) } \\
\hline$<55$ & 109 & 22.1 & 91 & 18.5 & 108 & 21.9 & 100 & 20.3 & 85 & 17.2 & 493 & 100.0 \\
\hline $55-65$ & 110 & 22.8 & 88 & 18.2 & 103 & 21.3 & 101 & 20.9 & 81 & 16.8 & 483 & 100.0 \\
\hline$>65$ & 54 & 17.2 & 65 & 20.7 & 61 & 19.4 & 64 & 20.4 & 70 & 22.3 & 314 & 100.0 \\
\hline \multicolumn{13}{|c|}{ Period of randomisation } \\
\hline 1991-1995 & 76 & 19.0 & 92 & 22.9 & 85 & 21.2 & 80 & 20.0 & 68 & 17.0 & 401 & 100 \\
\hline 1996-1999 & 197 & 22.2 & 152 & 17.1 & 187 & 21.0 & 185 & 20.8 & 168 & 18.9 & 889 & 100 \\
\hline \multicolumn{13}{|l|}{ FIGO stage } \\
\hline Stage I & 23 & 21.7 & 19 & 17.9 & 24 & 22.6 & 25 & 23.6 & 15 & 14.2 & 106 & 100 \\
\hline Stage II & 37 & 24.3 & 34 & 22.4 & 39 & 25.7 & 21 & 13.8 & 21 & 13.8 & 152 & 100 \\
\hline Stage III & 171 & 21.0 & 155 & 19.0 & 157 & 19.2 & 176 & 21.6 & 157 & 19.2 & 816 & 100 \\
\hline Stage IV & 42 & 19.4 & 36 & 16.7 & 52 & 24.1 & 43 & 19.9 & 43 & 19.9 & 216 & 100 \\
\hline Total & 273 & 21.2 & 244 & 18.9 & 272 & 21.1 & 265 & 20.5 & 236 & 18.3 & 1290 & 100.0 \\
\hline
\end{tabular}


Table 2. Relative survival (\%) at 1 and 5 years, by deprivation category, and fitted deprivation gap in survival

Women recruited to trials

General population

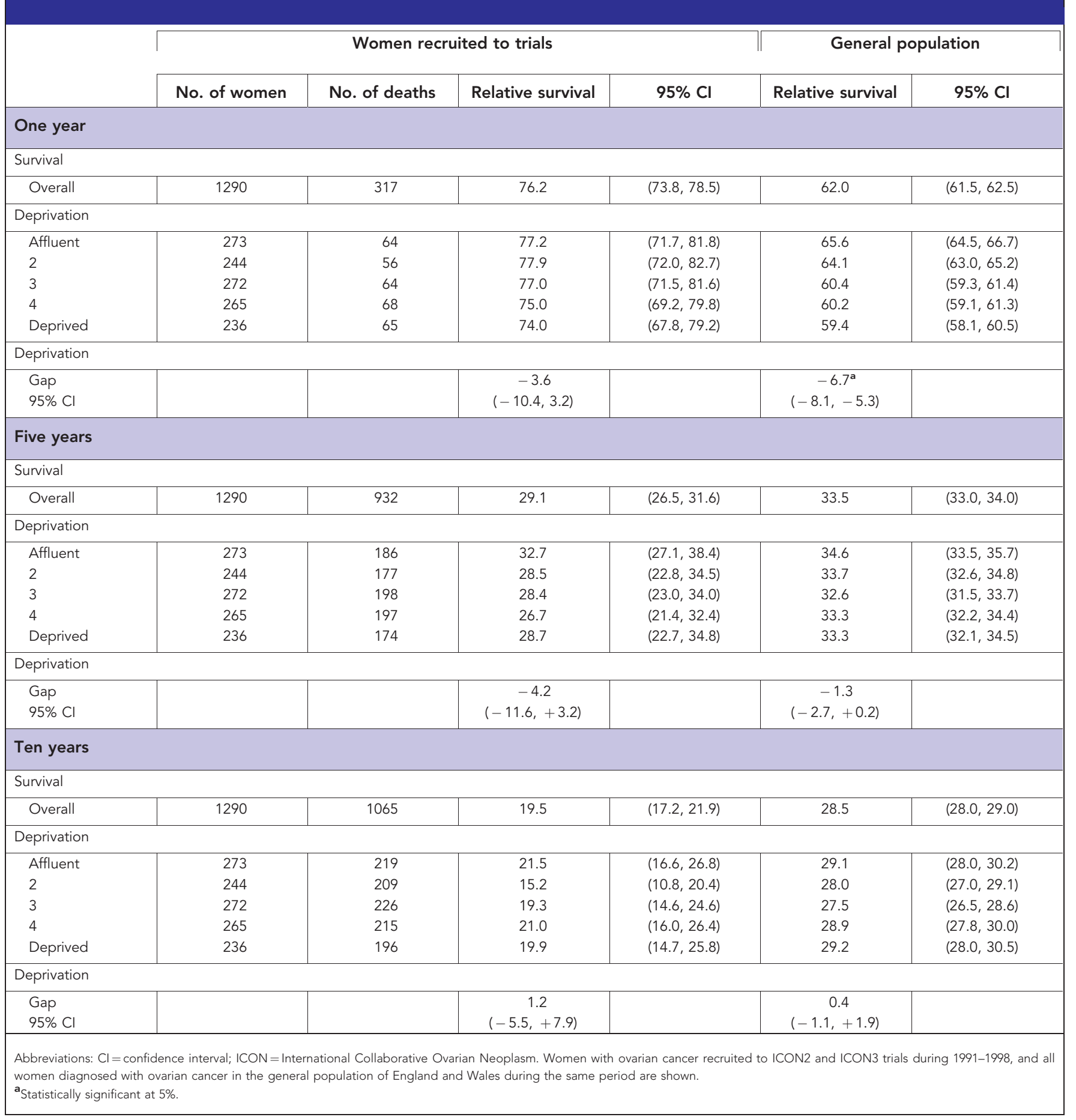

deprivation category with time since diagnosis was not statistically significant ( $P$-value 0.473 ), and none of the other interactions was found to be significant.

\section{DISCUSSION}

The distribution of socioeconomic status among the women recruited to these ovarian cancer trials in the 1990s was closely similar to that seen among women with ovarian cancer in the general population during the same period. This suggests that recruitment to the trials was carried out without socioeconomic bias. The socioeconomic status of the women was not known to the trial investigators: it was established only for our study, many years after closure of the trial, on the basis of each woman's small area of residence at diagnosis.

Misclassification of socioeconomic status by area of residence is likely to be very minor because the areas used are very small $(N=34378$, mean population 1500$)$ and they were designed to be fairly homogeneous for socioeconomic status. Since we used an ecological measure, any bias in the socioeconomic gradient in outcome arising from such misclassification would be towards the null, both for the trial patients and the general population.

We found no strong evidence of a gradient in 1-year, 5-year or 10 -year survival across five socioeconomic categories among 
women recruited to these two randomised trials of ovarian cancer treatment in England and Wales in the 1990s. This was at a time when a significant deprivation gap in 1-year ovarian cancer survival existed in the general population. Further, there was no statistically significant difference between rich and poor women in the excess hazard of death within 5 years in the trials, either before

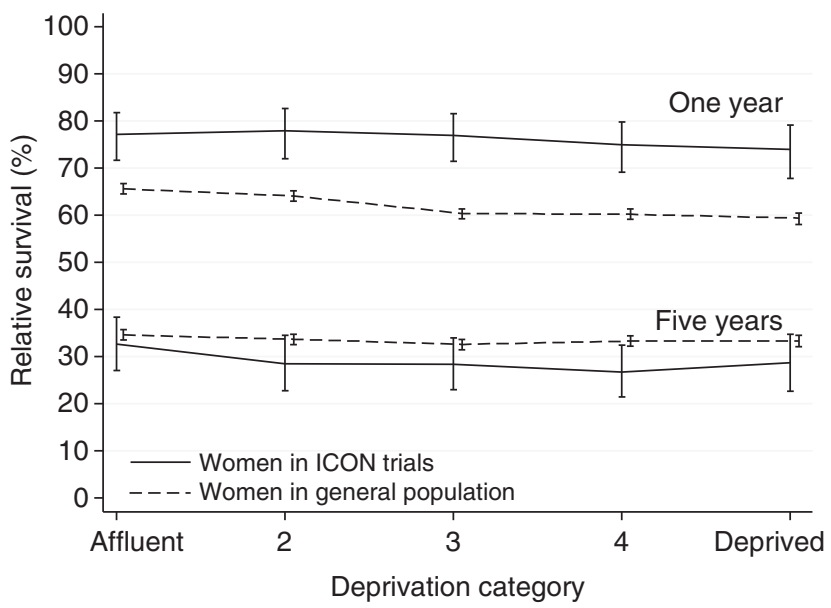

Figure 2. One- and five-year relative survival (\%) by deprivation category for women diagnosed with ovarian cancer during 1991-1998 in the ICON trials and in the general population of England and Wales. or after adjustment for age, calendar period and FIGO stage at diagnosis.

By contrast, for women with ovarian cancer in the general population, there was a significant deprivation gap in 1-year survival throughout the period 1971-1999 (Coleman et al, 1999). The absence of such a gradient among women recruited to randomised trials during the 1990s suggests that the socioeconomic differences in survival in the general population may be because of inequity of access to or quality of treatment.

Stage at diagnosis was similar for women in each deprivation category, and no deprivation gap in survival was observed in the trial. As survival does vary with deprivation in the general population, this suggests that deprived women in the general population are generally diagnosed at a more advanced stage than affluent women, and/or that there are socioeconomic differences in access to optimal treatment (Kitchener, 2008). Some evidence exists on stage: in a population-based audit in Scotland, the proportion of FIGO stage III/IV tumours was higher among women in the most deprived category than among those in the least deprived (67.3\% vs 58.2\%); the proportion of women for whom stage data were missing was also slightly higher $(11.9 \%$ vs 7.9\%; Brewster et al, 2001).

The deprivation gap in 5- and 10-year survival in the general population was not statistically significant for women diagnosed after 1990. This is consistent with the significant and persistent deprivation gap (around 5\%) in 1-year survival in England and Wales, but not at 5 or 10 years, throughout the period 1986-1999 (Coleman et al, 1999). Most of the excess deaths associated with

Table 3. Excess hazard ratios (EHR) of death within 5 years of diagnosis (with $95 \%$ confidence intervals) by deprivation category, before and after adjustment for age, calendar period of randomisation and FIGO stage at diagnosis

\begin{tabular}{|c|c|c|c|c|c|c|}
\hline & \multicolumn{3}{|c|}{ Unadjusted } & \multicolumn{3}{|c|}{$\begin{array}{c}\text { Adjusted for age and calendar period of } \\
\text { randomisation }\end{array}$} \\
\hline & \multicolumn{6}{|c|}{ Global } \\
\hline & EHR & $95 \% \mathrm{Cl}$ & $P$-value & EHR & $95 \% \mathrm{Cl}$ & $P$-value \\
\hline \multicolumn{7}{|c|}{ Deprivation } \\
\hline $\begin{array}{l}\text { Affluent } \\
2 \\
3 \\
4 \\
\text { Deprived }\end{array}$ & $\begin{array}{l}1.00 \\
1.06 \\
1.12 \\
1.20 \\
1.17\end{array}$ & $\begin{array}{l}(0.86-1.30) \\
(0.91-1.36) \\
(0.98-1.46) \\
(0.95-1.44)\end{array}$ & 0.391 & $\begin{array}{l}1.00 \\
1.02 \\
1.11 \\
1.14 \\
1.08\end{array}$ & $\begin{array}{l}(0.83-1.26) \\
(0.91-1.35) \\
(0.93-1.39) \\
(0.88-1.33)\end{array}$ & 0.707 \\
\hline \multicolumn{7}{|c|}{ Age at randomisation } \\
\hline $\begin{array}{l}<55 \text { Years } \\
55-65 \text { Years } \\
>65 \text { Years }\end{array}$ & & & & $\begin{array}{l}1.00 \\
1.25 \\
1.25\end{array}$ & $\begin{array}{l}(1.08-1.45) \\
(1.06-1.48)\end{array}$ & 0.005 \\
\hline \multicolumn{7}{|c|}{ Period of randomisation } \\
\hline $\begin{array}{l}1991-1995 \\
1996-1999\end{array}$ & & & & $\begin{array}{l}1.00 \\
0.85\end{array}$ & $(0.74-0.98)$ & 0.024 \\
\hline \multicolumn{7}{|c|}{ FIGO stage at diagnosis } \\
\hline \multirow[t]{3}{*}{$\begin{array}{l}\text { Stage I } \\
\text { Stage II } \\
\text { Stage III } \\
\text { Stage IV }\end{array}$} & & & & $\begin{array}{l}1.00 \\
1.12 \\
2.92 \\
4.30\end{array}$ & $\begin{array}{l}(0.76-1.65) \\
(2.13-4.00) \\
(3.07-6.01)\end{array}$ & $<0.001$ \\
\hline & \multicolumn{3}{|c|}{ Deviance $=1047$} & \multicolumn{3}{|c|}{ Deviance $=861$} \\
\hline & \multicolumn{3}{|c|}{ Residual degrees of freedom $=884$} & \multicolumn{3}{|c|}{ Residual degrees of freedom $=878$} \\
\hline
\end{tabular}


deprivation among women with ovarian cancer occur within 5 years of diagnosis. Ovarian cancer is often diagnosed at an advanced stage; therefore, many women will not receive treatment of curative intent, but palliative treatment such as second-line chemotherapy or surgery for recurrent or progressive disease may be less available to deprived women. The reduction in the deprivation gap in survival by 5 years after diagnosis may therefore reflect the inevitable course of disease. The absence of a deprivation gap in survival at 5 years and more may also be attributable to better access to specialist treatment for more affluent women (Kitchener, 2008). No socioeconomic difference in 5-year survival was seen in a study in Scotland (Shack et al, 2007).

Relative survival at 1 year among women with ovarian cancer in the ICON trials was $12-17 \%$ higher than among women in the general population, but 5- and 10-year survival were lower. Survival among patients recruited to clinical trials is often higher than for the generality of cancer patients because trial patients receive at least the best available treatment, adherence to protocol is strict and they are often younger than average, as was the case here. The lower long-term survival among women in the ICON trials therefore merits consideration. It is probably attributable to several selection factors: (a) FIGO stage III or IV disease was more common among women in the ICON trials (80\%) than in the general population $(60-70 \%)$; (b) women with ovarian tumours of borderline malignancy were not included in the trials, and these tumours generally have a good long-term prognosis; (c) women in the trials were fit enough to receive chemotherapy, and thus more likely to survive at least 1 year after diagnosis, but without longerterm benefit: median survival was 33 months in ICON2 and 36 months in ICON3. In short, women recruited to the ICON trials were not only younger than is typical for all women with ovarian cancer (median age 58 vs 65 years), but probably somewhat fitter, despite having more advanced disease.

The principal strength of this study is the follow-up of all the women for their vital status, many years after closure of the ICON trials in 1998. This allowed survival analysis up to 2007, by which time most of the women had died. Although follow-up in the trials was from the date of randomisation, not from the date of diagnosis, as in population survival studies, the median difference between diagnosis and randomisation was just 25 days. These were large trials; however, they were not designed to examine survival in five categories of socioeconomic deprivation. We can rule out a large deprivation gap in survival; however, statistical power to detect more modest effects of deprivation is limited. No information on stage was available in the population-based data to enable the same type of adjustment carried out with the trial data. Nonetheless, a generalised linear model that includes follow-up time, deprivation and age showed that deprivation was significantly related to survival (global likelihood ratio test $P$-value $<0.001$; results not shown).

The main limitation of this study is statistical power. Examination of survival differences by socioeconomic status was not part of the original trial designs; the trials would need to have been several times larger had that been so. We deliberately selected the largest available MRC trials of treatment for ovarian cancer. There are only moderate numbers of events (deaths) in each deprivation category. This study represents opportunistic secondary use of high-quality data from well-conducted randomised trials to examine a hypothesis about one possible cause of socioeconomic inequalities in cancer survival in the general population.

The ICON2 and ICON3 trials concerned primary treatment. The women in the trials were not a random sample of all women with ovarian cancer. They differed from all women with ovarian cancer in England and Wales in terms of age and stage of disease at diagnosis. These patterns help to explain the differences in overall survival between women with ovarian cancer in the trial and those in the general population. By contrast, the socioeconomic status of the women in the trials was remarkably similar to that of all women with ovarian cancer in England and Wales.

The women in these two well-conducted ovarian cancer trials were recruited with similar levels of disease, they were given treatment of equally efficacy and their socioeconomic status was not overtly known or recorded during the trials.

The absence of a socioeconomic gradient in short-term survival is therefore important. It strongly suggests that equal treatment of cancer patients can yield equal outcome, regardless of socioeconomic status. Given the persistent socioeconomic inequalities in survival among women with ovarian cancer in the general population, and among cancer patients more generally (Coleman et al, 1999; Rachet et al, 2010), that seems an observation worth making.

\section{CONCLUSION}

The findings showed no statistically significant association between socioeconomic deprivation and relative survival from ovarian cancer among women in two clinical trials whose socioeconomic status was not known at the time of the trial, and who were given treatments that, as it transpired, were of equal efficacy.

In other words, the trial produced evidence that equal treatment yields equal outcome, regardless of socioeconomic status.

The significant deprivation gap in 1-year relative survival seen among women with ovarian cancer in the general population may therefore be attributable to socioeconomic differences in access to optimal treatment.

\section{CONFLICT OF INTEREST}

The authors declare no conflict of interest.

CENTRES AND CLINICIANS PARTICIPATING IN ICON2 AND ICON3 IN ENGLAND AND WALES: FOR A FULL LIST OF INVESTIGATORS, SEE PARMAR et al $(1998,2002)$

Aberystwyth: Bronglais General Hospital (AT Axford); Bangor: Ysbyty Gwynedd (N Stuart); Birmingham Heartlands Hospital (I Fernando); Birmingham Women's Hospital (KK Chan, C Poole, ST Kehoe); Birmingham: City Hospital (C Poole, D Spooner); Birmingham: Queen Elizabeth Hospital (A Chetiyawardana, I Fernando, J Mould, C Poole, D Spooner); Bradford Royal Infirmary (C Bradley); Bristol Oncology Centre (A Brewster, S Falk, J Graham); Cambridge: Addenbrooke's Hospital (R Allerton, HM Earl, R Osborne, C Wilson, R Hawkins); Cardiff: Velindre Hospital (M Adams); Coventry: Walsgrave Hospital (M Hocking, C Irwin, A Stockdale); Cumberland Infirmary (J Nicoll); Derby City Hospital (IV Scott); Derbyshire Royal Infirmary (D Guthrie); Essex County Hospital (P Murray, S Tahir); Exeter: Royal Devon and Exeter Hospital (A Hong); Gateshead: Queen Elizabeth Hospital (N Bailey, J Bozzino, J Monaghan, A Lopes); Guildford: St Luke's Cancer Centre (A Folkes, R Laing, C Topham); Huddersfield Royal Infirmary (J Joffe); Ipswich Hospital (J Morgan); Jersey General Hospital (S Hima, C Williams); Lancaster: Royal Lancaster Infirmary (M McIllmurray); Leeds General Infirmary (E Buxton, KR Peel); Leeds: St James's University Hospital ( $\mathrm{T}$ Perren); Leicester Royal Infirmary (S Khanna, F Madden); Lincoln: County Hospital (E Murray); Liverpool: Royal Liverpool Hospital (J A Green, P Hendy-Ibbs); London: Hammersmith Hospital (H Thomas, C Vernon); London: Middlesex Hospital (A Cassoni, JA Lederman, RL Souhami); London: North Middlesex Hospital (N Davidson, S Davies, S Karp); 
London: Charing Cross Hospital (G Rustin, MJ Seckl, R Coombes); London: Chelsea and Westminster Hospital (R Phillips); London: Guy's Hospital (PG Harper); London: King's College Hospital (F Calman, HJ Dobbs); London: Royal Free Hospital (A Jones, J Ledermann); London: Royal London Hospital (C Gallagher); London: Royal Marsden Hospital (M Gore); London: St Bartholomew's Hospital (A Clayton-Jolly, C Gallagher, M Slevin); London: St George's (F Lofts, J Mansi); London: St Mary's Hospital (C Coulter); London: St Thomas's Hospital (R Beaney, F Calman); London: Whittington Hospital (JA Ledermann); Maidstone Hospital (J Summers, M O’Brien); Manchester: Christie Hospital (D Crowther, G Jayson, J Radford, R Welch, P Wilkinson); Manchester: Tameside General Hospital (W O Goldthorp, J K Roberts); Middlesbrough: South Cleveland Hospital (R Garry); Northwood: Mount Vernon Hospital (P Hoskin, G Rustin); Newcastle General Hospital (J Bozzino, H Calvert); Northampton General Hospital (D Levy, R Matthew, M Paterson, J Stewart); Nottingham City Hospital (S Chan); Oxford: Churchill Hospital (T Ganesan, B Lavery); Oxford: Manor Hospital (A Chetiyawardana); Peterborough District General Hospital (LT Tan); Plymouth General (F Daniel); Poole General Hospital (N Cowley, R Osborne, CJ Williams); Romford: Oldchurch Hospital (M Quigley); Royal Shrewsbury Hospital (S Awwad, R Gopal); Salisbury District (T Iveson); Sheffield: Weston Park Hospital (R Coleman, DJ Radstone, MJ Whipp); Southampton General Hospital (JMA Whitehouse, CJ Williams); Southampton: Royal South Hants Hospital (V Hall, TJ Iveson, P Simmonds, JMA Whitehouse, CJ Williams); Southend General Hospital (A Lamont, A Robinson, CW Trask); Stoke City General Hospital (AW Clubb); Stoke Mandeville Hospital (N Warner); Stoke: North Staffordshire Royal Infirmary (F Adab, CWE Redman, JE Scoble); Sussex Oncology Centre (D Murrell, G Newman, M Wilkins); Windsor: Princess Margaret Hospital (D Cole, J Cullimore); Wirral: Clatterbridge Centre for Oncology (JA Green); Wolverhampton: New Cross Hospital (R Allerton); Wycombe General Hospital (B Lavery).

\section{REFERENCES}

Barnholtz-Sloan JS, Tainsky MA, Abrams J, Severson RK, Qureshi F, Jacques SM, Levin N, Schwartz AG (2002) Ethnic differences in survival among women with ovarian carcinoma. Cancer $\mathbf{9 4}$ : 1886-1893.

Brewster DH, Thomson CS, Hole DJ, Black RJ, Stroner PL, Gillis CR (2001) Relation between socioeconomic status and tumour stage in patients with breast, colorectal, ovarian, and lung cancer: results from four national, population based studies. $\mathrm{Br}$ Med J 322: 830-831.

Cancer Research UK Cancer Survival Group (2004) Life Tables for England and Wales by Sex, Calendar Period, Region and Deprivation. London School of Hygiene and Tropical Medicine, London School of Hygiene \& Tropical Medicine: London.http://www.lshtm.ac.uk/eph/ncde/ cancersurvival/tools/index.html, accessed 16 Feburary 2009.

Cancer Research UK Cancer Survival Group (2010) strel Computer programme version 5.8 for Cancer Survival Analysis. London School of Hygiene and Tropical Medicine: London.http://www.lshtm.ac.uk/eph/ ncde/cancersurvival/tools/, accessed 28 July 2011.

Carstairs V (1995) Deprivation indices: their interpretation and use in relation to health. J Epidemiol Community Health 49(Suppl 2): 3-8.

Coleman MP, Babb P, Damiecki P, Grosclaude PC, Honjo S, Jones J, Knerer G, Pitard A, Quinn MJ, Sloggett A, De Stavola BL (1999) Cancer Survival Trends in England and Wales 1971-1995: Deprivation and NHS Region. (Studies on Medical and Population Subjects No. 61). The Stationery Office: London.

Coleman MP, Rachet B, Woods LM, Mitry E, Riga M, Cooper N, Quinn MJ, Brenner H, Estève J (2004) Trends and socio-economic inequalities in cancer survival in England and Wales up to 2001. Br J Cancer 90: 1367-1373.
Cooper N, Quinn MJ, Rachet B, Mitry E, Coleman MP (2008) Survival from cancer of the ovary in England and Wales up to 2001. Br J Cancer 99(Suppl 1): 70-72.

Dickman PW, Sloggett A, Hills M, Hakulinen T (2004) Regression models for relative survival. Stat Med 23: 51-64.

Ederer F, Axtell LM, Cutler SJ (1961) The relative survival: a statistical methodology. Natl Cancer Inst Monogr 6: 101-121.

Estève J, Benhamou E, Croasdale M, Raymond L (1990) Relative survival and the estimation of net survival: elements for further discussion. Stat Med 9: 529-538.

Grizzle JE, Starmer CF, Koch GG (1969) Analysis of categorical data by linear models. Biometrics 25: 489-504.

Jeffreys M, Sarfati D, Stevanovic V, Tobias M, Lewis C, Pearce N, Blakely T (2009) Socioeconomic inequalities in cancer survival in New Zealand: the role of extent of disease at diagnosis. Cancer Epid Biomarkers Prev 18: 915-921.

Kitchener HC (2008) Clinical commentary-survival from cancer of the ovary in England and Wales up to 2001. Br J Cancer 99(Suppl. 1): 73-74.

Maringe C, Walters S, Butler J, Coleman MP, Hacker N, Hanna L, Mosgaard BJ, Nordin A, Rosen B, Engholm G, Gjerstorff ML, Hatcher J, Borge Johannesen T, McGahan CE, Meechan D, Middleton R, Tracey E, Turner D, Richards MA, Rachet B. ICBP Module 1 Working Group (2012) Stage at diagnosis and ovarian cancer survival: evidence from the International Cancer Benchmarking Partnership. Gynecol Oncol 127: 75-82.

McCullagh P, Nelder JA (1999) Generalized Linear Models 2Chapman and Hall: Boca Raton, FL, USA.

Neighbourhood Renewal Unit (2004) The English Indices of Deprivation 2004 (revised). Office for the Deputy Prime Minister: London.

Nur U, Rachet B, Parmar MK, Sydes MR, Cooper N, Stenning S, Read G, Oliver T, Mason M, Coleman MP (2012) Socio-economic inequalities in testicular cancer survival within two clinical studies. Cancer Epidemiol 36: 217-221.

Nur U, Rachet B, Parmar MKB, Sydes M, Cooper N, Lepage C, Northover JMA, James R, Coleman MP. AXIS Collaborators (2008) No socioeconomic inequalities in colorectal cancer survival within a randomised clinical trial. Br J Cancer 99: 1923-1928.

Parmar MKB, Adams M, Balestrino M, Bertelsen K, Bonazzi C, Calvert H, Colombo N, Delaloye JF, Durando A, Guthrie D, Hagen B, Harper P, Mangioni C, Perren T, Poole C, Qian W, Rustin G, Sandercock J, Tumolo S, Torri V, Vecchione F (2002) Paclitaxel plus carboplatin versus standard chemotherapy with either single-agent carboplatin or cyclophosphamide, doxorubicin, and cisplatin in women with ovarian cancer: the ICON3 randomised trial. Lancet 360: 505-515.

Parmar MKB, Torri V, Bonaventura A, Bonazzi C, Colombo N, Delaloye JF, Marsoni S, Mangioni C, Sandercock J, Sessa C, Williams C, Tinazzi A, Flann M, Geiser K, Scorpiglione N, Stewart JF, Chaves J, Palmeiro E, Curtain A, McCormack T, Gennatas C, Marras F, Oppo TG, Balestrino M, Malzoni C, Malzoni M, Belli M, Geminiani ML, Crestani G, Monaco A, Vavala V, Piatto E, Barattini G, Fornara PG, Chetri MC, Santeufemia G, Artioli F, Carone D, Fanizza G, Trentadue R, Priolo D, Scollo P, Nigro SC, Petrina M, Mastrantonio P, Spanna GD, Zagni R, Belloni C, Colleoni R, Redaelli L, Cavagnini A, Di Costanzo G, Perroni D, Arienti S, Orfanotti G, Cantoni FM, Secli R, Bianchi A, Martinello R, Mollica G, Maizzi D, Picchiarelli ME, Fiorini G, Borsani M, Colombo E, Garsia S, Melgrati L, Paggi G, Brunenghi GM, Casini M, Isa L, Algeri R, Prozio G, Belfiore G, Angelini F, D'Aprile M, Moreschi M, Mauri ML, Natale N, Senzani FM, Pavanato G, Poggi G, Garuti G, Luerti M, Cruciani G, Pagano F, Baccolo M, Poddi ER, Bocciolone L, Sabelli MA, Maggi R, Restelli C, D'Antona A, Locatelli MC, Pessi A, Raina A, Chiari S, Gabriele A, Pittelli MR, Iacobelli P, Dogliotti L, Gorzegno G, Musso P, Vegna G, Coco G, Alletti DG, Picciotto F, Lucchese V, Epis A, di Palumbo VS, Drudi G, Ravaioli A, Zampella D, Morandi MG, Gorga G, Zucchelli C, Cariello S, Galletto L, Sussio M, Massacesi L, Massacesi M, Carli A, Tucci E, Tajani E, Corrado G, Bumma S, Durando A, Massobrio M, Sberveglieri M, Biasio M, Guercio E, Jura R, Danese S, Wierdis T, Farnelli C, Tarantino G, Grassi R, Repetti F, Rocchi B, Grampa M, Ercoli A, Griso C, Signori E, Zanini L, Presti M, Klimek M, Urbanski K, Biswas A, Viegas O, Kochli O, Dreher E, Fey M, Beck G, Ludin J, Bonnefoi H, Krauer F, Bauer J, Delmore G, Furrer C, Lorenz U, Thurlimann B, Lorenz U, Bronz L, Sanna P, Wyss D, Goldhirsch A, Gyr T, Leidi L, Pastorelli G, Pagani O, Rey P, Hailer U, Benz J, Kaye SB, Reed NS, Symonds RP, Atkinson RJ, Axford AT, Rustin G, Seckl MJ, Scott IV Green JA, Guthrie D, Harper PG, Calman F, Dobbs HJ, Weir P, Cassoni A, Lederman JA, Souhami RL, Bozzino J, Adab F, Redman CWE, Scoble JE, Paterson M, Daniel F, Cowley N, Williams CJ, Spooner D, Hong A, McIllmurray M, Hendy-Ibbs P, Hall V, Iveson TJ, JMA Whitehouse, Garry R, 
Lamont A, Robinson A, Trask CW, Clubb AW, Murrell D, Newman G, Wilkins M, Goldthorp WO, Roberts JK, Radstone DJ, Whipp MJ,

Ledermann JA, Pater J, Buyse M, Omura G (1998) ICON2: randomised trial of single-agent carboplatin against three-drug combination of CAP (cyclophosphamide, doxorubicin, and cisplatin) in women with ovarian cancer. Lancet 352: 1571-1576.

Pocock SJ, Simon R (1975) Sequential treatment assignment with balancing for prognostic factors in the controlled clinical trial. Biometrics 31: 103-115.

Rachet B, Ellis L, Maringe C, Nur U, Chu T, Quaresma M, Shah A, Walters S, Woods LM, Forman D, Coleman MP (2010) Socioeconomic inequalities in cancer survival in England after the NHS Cancer Plan. Br J Cancer 103: 446-453.

Rachet B, Woods LM, Mitry E, Riga M, Cooper C, Quinn MJ, Steward JA, Brenner H, Estève J, Sullivan R, Coleman MP (2008) Cancer survival in England and Wales at the end of the 20th century. Br J Cancer 99(Suppl. 1): 2-10.

Shack LG, Rachet B, Brewster DH, Coleman MP (2007) Socioeconomic inequalities in cancer survival in Scotland 1986-2000. Br J Cancer 97: 999-1004.
StataCorp (2006) STATA statistical software. College Station. Stata Corporation: Texas.

Walters S, Maringe C, Butler J, Brierley JD, Rachet B, Coleman MP (2013) Comparability of stage data in cancer registries in six countries: lessons from the International Cancer Benchmarking Partnership. Int J Cancer 132: 676-685.

Woods LM, Rachet B, Coleman MP (2005) Choice of geographic unit influences socioeconomic inequalities in breast cancer survival. Br J Cancer 92: 1279-1282.

Woods LM, Rachet B, Coleman MP (2006) Origins of socioeconomic inequalities in cancer survival: a review. Ann Oncol 17: 5-19.

This work is published under the standard license to publish agreement. After 12 months the work will become freely available and the license terms will switch to a Creative Commons AttributionNonCommercial-Share Alike 3.0 Unported License. 